\title{
3 Research Square

\section{Social Compliance During High Stringency Periods Efficiently Reduces COVID-19 Incidence: Evidence from Google Mobility Reports}

Lasse Hyldig Hansen ( $\sim$ Lassehyldigh@gmail.com )

Aarhus University

Thomas Lykke Rasmussen

Aarhus University

Palle Villesen

Aarhus University

\section{Research Article}

Keywords: Social Compliance, High Stringency Periods, Reduces COVID-19 Incidence, Google Mobility Reports, global Sars-CoV-2 pandemic

Posted Date: May 13th, 2021

DOI: https://doi.org/10.21203/rs.3.rs-501561/v1

License: (c) (i) This work is licensed under a Creative Commons Attribution 4.0 International License. Read Full License 


\section{Social Compliance During High Stringency Periods Efficiently Reduces COVID-19 Incidence: Evidence from Google Mobility Reports}

Lasse Hyldig Hansen $\left.{ }^{1}{ }^{*}\right)$, Thomas Lykke Rasmussen ${ }^{2} \&$ Palle Villesen ${ }^{3,4}$

1) School of Communication and Culture, Aarhus University, DK-8000 Aarhus $C$

2 ) Department of Mathematics, Aarhus University, DK-8000 Aarhus C

3) Bioinformatics Research Centre, Aarhus University, DK-8000 Aarhus C

4) Department of Clinical Medicine, Aarhus University, DK-8200 Aarhus N

\section{Abstract}

It is crucial to understand and learn as much as possible from the current global Sars-CoV-2 pandemic for the sake of future precautions. Apart from strong government restrictions such as complete lockdowns, curfews, and mask mandates, other factors influence viral transmission.

Since June 2020, Denmark has had an extensive test and surveillance program and made data publicly available at the municipality level. Here we use these data and integrate publicly available data on government restrictions, weather data, and mobility data to model COVID-19 incidence in 98 Danish municipalities from September 2020 to February 2021.

The inclusion of municipality heterogeneity, weather and mobility data increases the amount of variance explained by $\sim 29 \%$ compared to a simpler model taking only incidence and restrictions into account. We found a strong and significant effect from temperature which interacts with government restrictions. Our results indicate that higher temperatures limit viral transmission when government restrictions are low, but that the temperature effect diminishes under stronger restrictions. This is most likely due to a change in human behavior rather than a biological effect.

Likewise, we found that changes in residential mobility were significant factors that also interacted with restrictions. When restrictions were strong, we found that increased residential mobility resulted in decreased COVID-19 incidence, suggesting residential mobility as a proxy for compliance.

Our results show the increased explanatory power of integrating different variables when modeling COVID-19 incidence. The weather seems to predict human behavior in a quite predictable way and mobility data could be used to measure current compliance with government restrictions. 


\section{Introduction}

Cases of COVID-19 were first reported from China in 2019, and within a few months, it became a pandemic that challenged all aspects of human life. As of April 2021, more than 130 million people have been infected with the disease worldwide, and the outbreak has caused millions of deaths. In Europe, most countries experienced a first wave of cases in spring 2020 resulting in national lockdowns, travel bans, and public restrictions. Incidence rates decreased and restrictions were lifted during the summer of 2020. The second wave hit most countries during the fall of 2020 , and many countries were again forced to impose national lockdowns, travel bans, and other measures.

In Denmark, the first wave led to a national lockdown and several public restrictions that have since been revised numerous times based on the basic reproduction number. The restrictions involve closings of, among others, restaurants, schools, and retail stores as well as mandatory facemasks at e.g. transit hubs and grocery stores.

The fast intervention of the Danish government has been mentioned as the main reason for the fact that the stringency of the restrictions in Denmark has, compared to other countries, proved to be very effective (Dergiades et al., 2020).

Denmark has since 12 May 2020 offered free PCR tests to both symptomatic and asymptomatic individuals, and the testing capacity has since been increased enormously. Denmark has conducted more than 25 million tests, making Denmark one of the most frequently tested nations in Europe per capita (SSI, n.d.). Currently (April 2021), Denmark is testing $>5 \%$ of the population every day ( $+400,000$ tests daily, population size 5.8 mio). This extensive test and surveillance program has confirmed more than 240,000 cases of COVID-19.

Countries have sought to control COVID-19 transmission by imposing restrictions on the population as well as through other social distancing campaigns. Strict restrictions have in literature been associated with economic and social consequences. For instance, a lockdown has been associated with a greater degree of loneliness, (Varga et al., 2021) a decrease in production, and a lack of sales and activity in many industries (Pak et al., 2020). On the other hand, existing literature has found that government restriction measures have been a valuable tool in reducing transmission (Qiu et al., 2020). Adding to this, studies have found that travel restrictions are important measures in delaying the spread of a pandemic (Chinazzi et al., 2020). The travel quarantine in Wuhan delayed the overall epidemic increase in the country by two days in Mainland China. The same effect was found from public transportation strikes (Godzinski \& Suarez Castillo, 2019).

Literature reports that weather variables also help to increase and bring down pandemics (Lowen et al., 2007). Lowen et al. (2007) found that warmer temperatures help reduce the prevalence of viruses. The presumed effect of weather variables on the facilitation of the spread of SARS was substantiated by (Chan et al., 2011a). Additionally, evidence suggests that different demographic, socioeconomic, and mobility trends have an impact on the transmission of COVID-19 (Badr et al., 2020; Chinazzi et al., 2020; Qiu et al., 2020). Furthermore, Yilmazkuday (2020) and Nouvellet et al. (2021) report that lowered mobility, 
from Google mobility reports, is associated with fewer COVID-19 cases. Of other, relevant literature, Borkowski et al. (2021) report that individual travel time is reduced by $66 \%$ across all age groups, suggesting that the social dynamics of citizens during a pandemic changes.

As a consequence of the aforementioned, it is of great importance to understand the association between social dynamics, socioeconomic demographics, weather variables, and the transmission of COVID-19. Uncovering the relationship between these variables and government restrictions will be a useful tool in guiding political rhetoric and in avoiding the spread of misinformation that can potentially fuel polarity in the population. Also, understanding the effect of restrictions and their interaction with weather and mobility changes in society can help to ensure that social distancing is not done in vain. Mobility trends will add to this picture by identifying how mobility in society affects virus transmission and will help guide how and when restrictions must be imposed. Furthermore, it will help understand what thrives a pandemic, which will be useful to contain transmission rates.

We have implemented a mixed-effects model to understand the transmission of COVID-19 in Denmark. This is accomplished by modeling daily COVID-19 incidence (cases per 100,000 residents) on each day in each of Denmark's 98 municipalities. We use stringency conditions, Google mobility data (Google, n.d.), DMI weather data (DMI, n.d.), and municipality demographic data together with publicly available COVID-19 infection data as fixed/explanatory variables. Specifically, the model considers the percentage changes in mobility around residential areas and transit stations compared to a baseline. Therefore, an increase in residential mobility indicates that people are staying more at home, and an increase in transit mobility indicates that people are using more public transportation. Furthermore, the model considers the daily average temperature and precipitation in each municipality. Of demographic variables, the proportion of young adults and the share of buildings and urban areas per capita are hypothesized to affect transmission rates. When the share of buildings and urban areas per capita is small, it follows that there are few buildings per person. Thus, people are living more densely packed, and are therefore more exposed to the spread of viruses. The model is autoregressive and considers the incidence of COVID-19 with a lag of nine days as an explanatory variable in accordance with Backer et al. (2020). All other time-variant variables are lagged with nine days in order to determine the time-dependent effect of the fixed effects on the incidence in the present. Furthermore, the effect of previous incidence, mobility variables, and weather variables are considered to interact with the stringency index in Denmark at each given time.

The reason for implementing a mixed-effects model is twofold; firstly, to account for the variability in the COVID-19 incidence for the different municipalities. Secondly, to account for the heterogeneity between individual days throughout the period. Therefore, we allow the model to have varying effects for both days and municipalities. This paper contributes to the existing literature by elucidating the relationship between weather variables, previous incidence, mobility data, and COVID-19 incidence in the Danish municipalities. Moreover, our model takes into consideration how these dynamics are dependent on the stringency condition at the time in Denmark. 


\section{Results}

\section{Overall Model Fit}

The complete derivation of the mixed-effects model is described in the methods section, the complete model is given in equation (6). To deem this model relevant in explaining COVID-19 cases per 100,000 capita in the 98 municipalities of Denmark, we start by considering the fit of the model. When determining model-fit in mixed-effects models, a different route than the usual for linear models is taken into consideration. In accordance with Nakagawa \& Schielzeth (2013), we calculate the marginal and conditional $R^{2}$ for our model of choice. We get that the Marginal $R^{2}=0.295$. This measure comprises the proportion of variability our fixed effects explain. The measure is derived by dividing the variance of the fixed effects by the total variability of the model. Furthermore, we get that the Conditional $R^{2}=0.861$.This suggests that $86.1 \%$ of the variance in the data is explained by both fixed and random/varying effects from our model.

To evaluate the mixed-effects model and understand the information cost/benefit of adding more predictors and varying effects, we use model comparison. The null model that we will consider for model comparison is the simple model where the independent variables are cases per 100,000 capita with a lag of nine days and its interaction with stringency. Through a likelihood ratio test, we established that our model predicts the outcome better than the null model: $\chi^{2}(12)=24350, p<.001$ (table 1 ). The null model has an Adjusted $R^{2}=0.572$, which means it explains $57.2 \%$ of the variance of the independent variable. Akaike Information Criteria value for the varying effects model is also lower, which is an enforcement of the validity of the independent variables and varying effects chosen. Generally, the mixed-effects model explains daily COVID-19 incidence to better proximity, which is observed for most municipalities (figure 1 and $\mathrm{S} 1$ ).

Table 1. Model comparison results.

\begin{tabular}{|c|c|c|c|c|c|c|c|c|}
\hline Model & npar & AIC & BIC & logLik & deviance & $\chi^{2}$ & Df & $\operatorname{Pr}(>$ Chisq $)$ \\
\hline Cases $\sim$ (Cases $\times$ Stringency) & 5 & 128477 & 128516 & -64234 & 128467 & & & \\
\hline Mixed effects model & 17 & 104151 & 104283 & -52058 & 104117 & 24350 & 12 & $<0.0000$ \\
\hline
\end{tabular}

\section{Strong effect of previous incidence and its interaction with stringency}

We found that the lagged incidence had a strong, positive, and significant effect on the incidence in the present: $\beta_{1}=5.603 \pm 0.1081$ (s. e.m.), $t(17321.3)=51.850, p<0.001$ (table 2) This suggests that COVID-19 incidence has a strong positive autoregressive relationship with COVID-19 nine days prior when stringency is on average for the period (grey line in S2). 
Furthermore, the relationship between present and lagged incidence had a significant negative interaction with government stringency index (nine days prior to the dependent variable): $\beta_{9}=-1.1624 \pm 0.1116$ (s.e.m.), $t(17293.5$ ) $=-10.417, p<0.001$ (table 2) .

This suggests that the effect of incidence lagged on incidence in the present significantly decreases when stringency goes from average to 1 standard deviation above average. The effect implies that present and future COVID-19 incidence is strongly correlated. However, it also implies that the effect is weaker under more strict restrictions (supl. figure 2). Not surprisingly, the same incidence leads to more cases nine days later under looser restrictions. This clearly demonstrates that restrictions work in Denmark.

\section{Strong Effect of Temperature under Low Stringency Conditions}

Temperature with a lag of nine days had a significant, negative, main effect on cases per 100,000 capita: $\beta_{4}=-1.9078 \pm 0.5261$ (s. e.m.), $t(1305.5$ ) $=-3.626, p<0.001$ (table

2). This suggests that higher temperatures were followed by lower amounts of COVID-19 cases per 100,000 nine days after, at average government stringency (grey line in figure 2A). Furthermore, the effect of temperature nine days ago on incidence to the present had a significant positive interaction effect with government stringency (nine days prior to the dependent variable) $\beta_{11}=2.8518 \pm 0.4551$ (s.e.m.), $t(5693.5)=6.266, p<0.001$ (table

2). This demonstrates that the effect of stringency on incidence in the present significantly increases when stringency goes from average to 1 standard deviation above average. Hence, when government restrictions are more intruding, the negative effect of temperature on incidence diminishes. However, the interaction effect (figure 2A) also displays the importance of understanding the relationship between temperature and stringency on the COVID-19 incidence. In sum, when stringency is low, higher temperatures result in fewer incidences (yellow line in figure 2A). However, when stringency is high, the effect of temperature on incidence diminishes (blue line in figure $2 \mathrm{~A}$ ).

\section{Strong Effect of Residential Mobility Change under High Stringency Conditions}

Residential mobility changes with a lag of nine days had a significant, negative, main effect on cases per 100,000 capita:

$\beta_{6}=-1.5479 \pm 0.3304$ (s.e.m.), $t(11600.3)=-4.684, p<0.001$ (table 2). Under average restrictions, the increase of mobility around residential areas leads to less COVID-19 incidences nine days later. Furthermore, the relationship between incidence in the present and change in residential mobility has a significant interaction effect with stringency index: $\beta_{13}=-1.6108 \pm 0.2538$ (s.e.m.), $t(14735.6)=-6.346, p<0.001$ (table 2). The interaction indicates that the negative effect of residential mobility changes (nine days prior) on COVID-19 incidence increases when the stringency index goes from average to 1 standard deviation above average. This suggests that in high stringency conditions (blue line in figure $2 \mathrm{~B}$ ), with increased mobility around residential areas, the incidence is lower nine days later. The opposite is true, when residential mobility decreases, in periods with high stringency, which results in higher incidence nine days later. When stringency is low (1 SD below average), the change of mobility around residential areas does not impact incidence nine days after (yellow line in figure 2B). The effect is still present when stringency is on average (grey line in figure $2 \mathrm{~B}$ ), where mobility increase in residential areas results in lower COVID-19 incidence. 
Table 2 Summary statistics of the mixed effect model on incidence of COVID-19

\begin{tabular}{|c|c|c|c|c|}
\hline Coefficient & Estimate & Std. E. & t-stat & p-value \\
\hline incidence $_{i, t-9}$ & 5.603 & 0.1081 & 51.850 & 0.0000 \\
\hline stringency $_{t-9}$ & 0.7810 & 0.4906 & 1.592 & 0.1115 \\
\hline transit $_{r, t-9}$ & 0.6118 & 0.1544 & 3.961 & 0.0001 \\
\hline residential $_{r, t-9}$ & -1.5479 & 0.3304 & -4.684 & 0.0000 \\
\hline temperature $_{i, t-9}$ & -1.9078 & 0.5261 & -3.626 & 0.0003 \\
\hline precipitation $_{i, t-9}$ & 0.1436 & 0.1126 & 1.275 & 0.2022 \\
\hline${\text { builded } \text { areas }_{i}}$ & -0.6642 & 0.2552 & -2.603 & 0.0108 \\
\hline young adults $_{i}$ & -0.3717 & 0.2552 & -1.457 & 0.1486 \\
\hline$(\text { incidence } \times \text { stringency })_{i, t-9}$ & -1.1624 & 0.1116 & -10.417 & 0.0000 \\
\hline$(\text { transit } \times \text { stringency })_{i, t-9}$ & -0.4284 & 0.1311 & -3.269 & 0.0000 \\
\hline$(\text { residential } \times \text { stringency })_{i, t-}$ & -1.6108 & 0.2538 & -6.346 & 0.0000 \\
\hline$(\text { temperature } \times \text { stringency })_{i}$ & 2.8518 & 0.4551 & 6.266 & 0.0000 \\
\hline$(\text { precipitation } \times \text { stringency })_{i}$ & 0.3303 & 0.1102 & 2.996 & 0.0027 \\
\hline
\end{tabular}




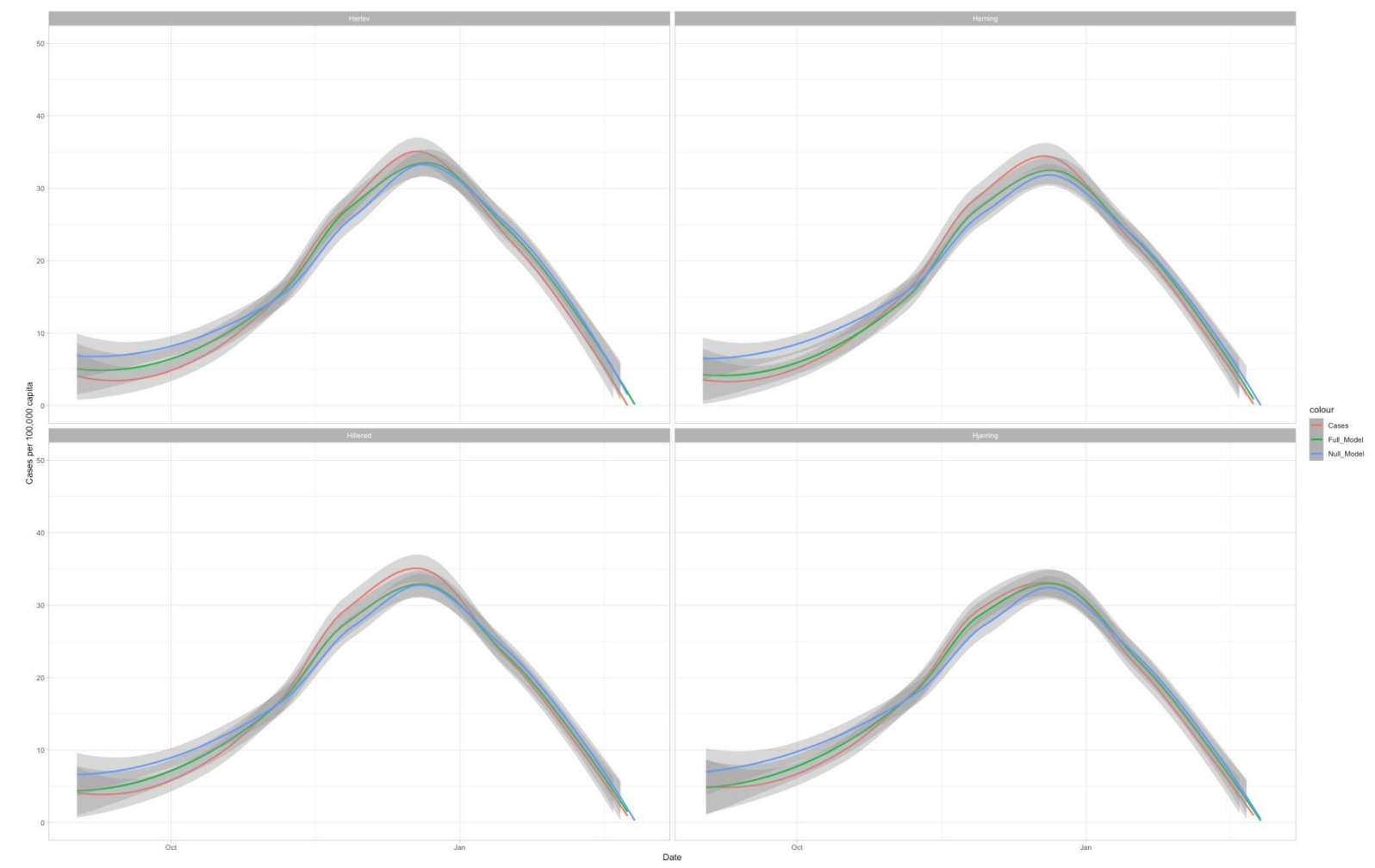

Figure 1. Observed and predicted COVID-19 incidence (smoothed cases per 100,000 people) for four Danish municipalities in the period of analysis. Observed incidence (red line), Null model predictions (blue line) and mixed effects model predictions (green line). The grey shading around the lines is the $95 \%$ confidence interval.

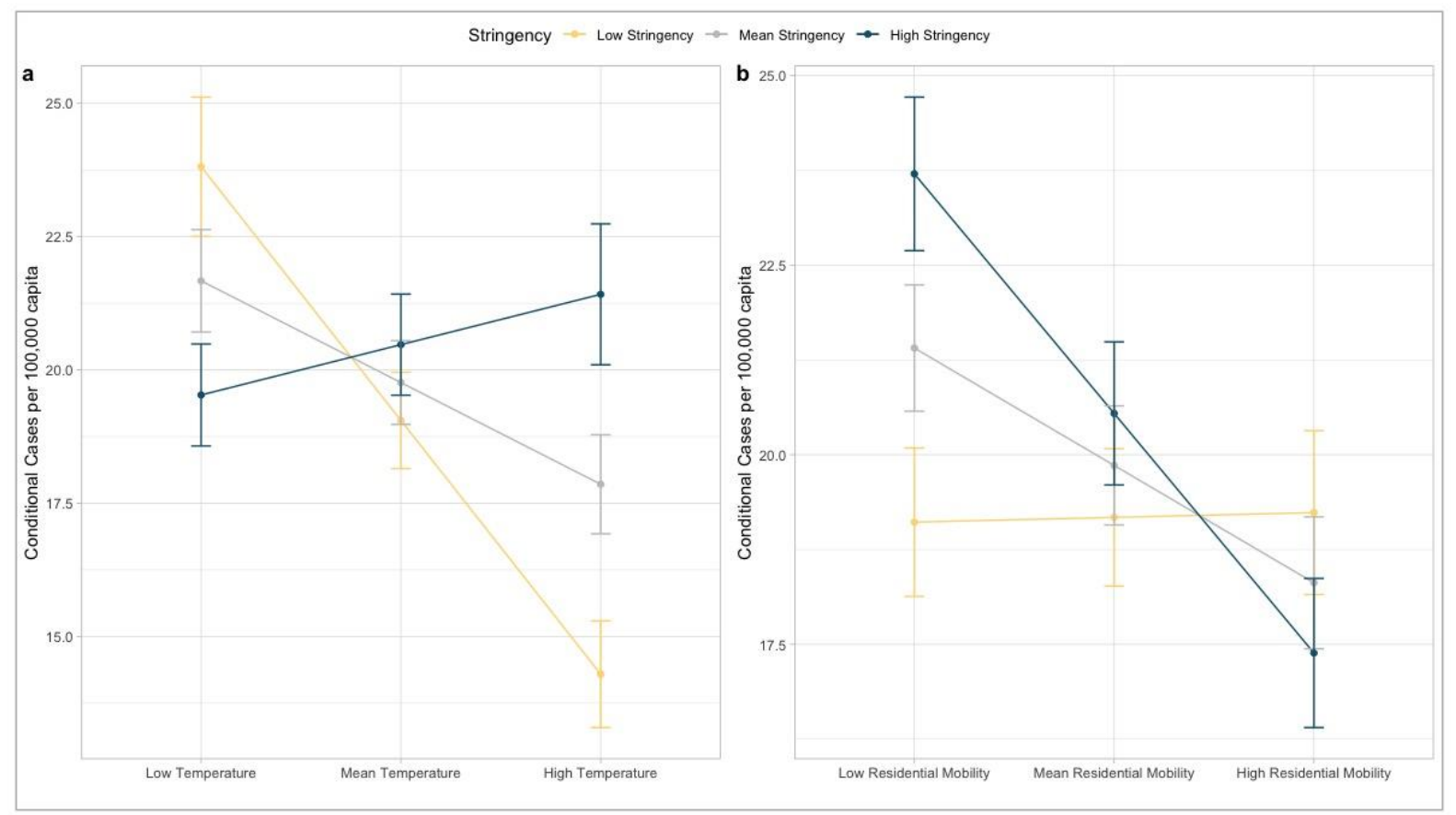


Figure 2. Conditional interaction plots of two of the interaction effects from the model. Figure a: the interaction effect of temperature on incidence that depends on the stringency index. Figure $b$ : the interaction effect of residential mobility change on incidence that depends on the stringency index. Temperature and residential mobility change are on a z-scale, and varies between -1 (1 SD below average) and 1 (1 SD above average). The same is the case for stringency, where 3 conditions of stringency are visualized (1 SD below average, average, and 1 SD above average). The $y$-variable is the conditional average COVID-19 incidence given the parameters of the model (predicted values given our model).

\section{Discussion}

How do societal and environmental impacts affect the number of COVID-19 cases in Denmark? This study imposes a mixed-effects model to understand the effect of mobility patterns, weather variables, and municipality demographics on the transmission of COVID-19.

We found strong evidence of COVID-19 transmission being affected by temperature in Denmark. In general, we saw that higher temperatures result in fewer COVID-19 incidences. Surprisingly, we found a significant interaction between temperature and stringency conditions in Denmark. Our results show that temperature didn't have a huge impact on virus transmission in high stringency periods. This could be due to a well-implemented strategy of interventions by the Danish government to contain the virus, making any weather effect negligible. However, under low stringency conditions, we found that temperature was an important factor that seemed to keep virus transmission at a low level. This could potentially be due to the stability of the virus losing viability in higher temperatures as suggested by (Chan et al., 2011). Alternatively, it could simply be by virtue of the social dynamics of the Danish citizens changing with higher temperatures (e.g. less indoor activities following higher temperatures). We speculate that the most likely explanation is a change in behavior since countries with more sunlight than Denmark (most countries) have experienced a similar Sars-CoV-2 outbreak. No matter the exact cause of this effect, higher temperatures prove to be an essential factor in decreasing virus transmission in Denmark.

Based on the newly released data from Google mobility reports, we were able to understand how transit and residential mobility in Denmark affected the incidence throughout the period of analysis. Our results showed a strong interaction between the mobility in the citizens of Denmark and stringency conditions imposed by the Danish government. Firstly, we found that people in Denmark differed in how they moved around transit and residential areas depending on imposed government restrictions. Essentially, citizens changed behavior with changing government restrictions. Secondly, we found that government-imposed restrictions affected COVID-19 incidence through this mobility. Generally, we found increased residential mobility (staying more at home) led to lower COVID-19 incidence (figure 2B). It is clear that when stringency is high and people stay at home (increased residential mobility), incidences of COVID-19 are reduced. The simple interpretation is that residential mobility reflects the level of compliance with government restrictions. Reduced residential mobility in times of high stringency was associated with more cases of COVID-19 per 100,000, while transmission in low stringency periods didn't depend on the change in residential mobility. The significant interaction effect could speak to the very high government trust reported in 
Denmark when compared to other countries (Petersen \& Roepstorff, 2021) as it indicates that Danish citizens are conforming to the restrictions.

Our mixed-effects model contained an autoregressive coefficient which was cases per 100,000 nine days prior to the present. The autoregressive coefficient and its interaction with stringency elucidate the importance of fast intervention in the COVID-19 pandemic. Intuitively, the best way to control the incidence is by reducing transmission. When Denmark saw an exponential rise in cases, stronger restrictions were quickly imposed. The autoregressive coefficient had a very strong impact on COVID-19 incidence to the present, which makes sense, when considering a pandemic that spreads from nose-mouth secretions (WHO, 2020). This result is also in accordance with (Dergiades et al., 2020) that suggests that early government interventions comprise the most efficient way of slowing down or reversing the growth rate of COVID-19.

Our model should not be seen as a model that can predict individual outbreaks in different municipalities as making such predictions is beyond the scope of the work, and thereby the model's predictive ability. Instead, our main aim was to understand overall effects and interactions and at the same time filter out the variance of the individual municipalities and dates.

\section{Methods}

We used data from the 98 municipalities of Denmark, restricted to the time span from 1st September 2020 when the test capacity in Denmark was sufficiently large for analysis until the end of February 2021. In these months, we avoided most of the local restrictions and lockdowns imposed during the start of the fall. This was necessary since the stringency index was only available at the national scale.

Time series of COVID-19 cases from the individual municipalities were scraped from the Danish Health Authority (SSI, n.d.).

We used an autoregressive linear mixed-effects model to understand the effect of changes in various relevant socioeconomic and social distancing factors on the incidence of COVID-19. The incidence was calculated as the share of cases per 100,000 residents in each municipality.

The variables that we hypothesized to influence COVID-19 incidence were all set to a lag of nine days in accordance with Backer et al. (2020) who found that social distancing influences new infections optimally at a 9-12 day lag. This assumes that the model can account for both incubation time and the time it takes for a person to be tested.

COVID-19 spreads between people via mouth and nose secretions (WHO, 2020). This indicates that the current incidence is serially correlated with the lagged incidence. Therefore, the amount of cases per 100,000, nine days prior to the dependent variable, is hypothesized to have an impact on the incidence in the present. We used the Oxford COVID-19 Government Response Tracker index to model Government restrictions (Hale et 
al., 2021). The index is calculated based on various types of government regularization such as school closings, cancellations of public events, contact tracing, and facial coverings.

Equation (1):

$$
\text { incidence }_{i, t}=\beta_{0}+\beta_{1}^{\text {incidence }_{i, t-9}}+\beta_{2} \text { stringency }_{t-9}+\epsilon_{i, t}
$$

We included two weather variables that are hypothesized to be linked to virus transmission; temperature and precipitation from all DMI owned stations in Denmark. The data were scraped from the DMI Meteorological Observation (metObs) API service and all DMI stations were assigned to the municipality in closest proximity (DMI, n.d.).

Equation (2):

$$
\text { incidence }_{i, t}=\beta_{3} \text { precipitation }_{i, t-9}+\beta_{4} \text { temperature }_{i, t-9}+\epsilon_{i, t}
$$

Furthermore, we considered the effect of different mobility changes from their baseline in Transit Stations and places of residence. Mobility data reports in Denmark were downloaded from COVID-19 Community Mobility Reports by Google (Google, n.d.). When data was present for the individual municipalities, mobility data was assigned by municipality level. However, if a given municipality had missing data for the given date, mobility data were assigned to each municipality at the region level. A total of 333 data points were removed because of missing data on both the municipality and region level (Rose, n.d.).

Equation (3):

$$
\text { incidence }_{i, t}=\beta_{5} \text { transit }_{r, t-9}+\beta_{6} \text { residential }_{r, t-9}+\epsilon_{i, t}
$$

Demographic differences between the municipalities were also hypothesized to have a significant effect on the spread of the virus. Specifically, we included the share of buildings and urban areas per capita ("buildings") as well as the percentage of people between the age of 19 and 29 ("youngadults") (Statistics Denmark, 2018, 2020). The share of buildings and urban areas per capita are assumed to be variables that describe how close people live in a given city. These variables are scraped from 'Danish Statistics', with an $\mathrm{R}$ integration package (Rose, n.d.).

Equation (4):

$$
\text { incidence }_{i, t}=\beta_{7} \text { building }_{i}+\beta_{8} \text { youngadults }_{i}+\epsilon_{i, t}
$$

The social dynamics of the residents in Denmark were hypothesized to change with different stringency conditions. Therefore, the stringency conditions, stringency ${ }_{i, t-9}$ is hypothesized to have an interaction effect with both the lagged incidence, the weather variables, and the mobility variables on the incidence in the present. These variables are hypothesized to differ based on the stringency index in Denmark through the time period, and hence impact social dynamics in different ways under different stringency conditions.

Equation (5): 


$$
\begin{array}{r}
\text { incidence }_{i, t}=\beta_{9}(\text { incidence } \times \text { stringency })_{i, t-9}+\sum_{j=10}^{11} \beta_{j}(\text { weather } \times \text { stringency })_{i, t-9} \\
+\sum_{k=12}^{13} \beta_{k}(\text { mobility } \times \text { stringency })_{i, t-9}+\epsilon_{i, t}
\end{array}
$$

We assume that demographic variables do not interact with the stringency index, since the demographic variables are not dynamic by themselves. The social dynamics that affect the COVID-19 incidence in the different municipalities should be captured by the interaction terms, and we only test for the main effect of demographic differences on COVID-19 incidence.

All explanatory variables were standardized, before conducting the analysis, by subtracting the mean of the variable and dividing by the standard deviation. Our variables of interest lie on different scales of magnitude, and the standardization allows for the comparison of scores between different variables. We kept the response as cases per 100,000 residents on its original scale to make the relationship between the coefficients and transmission of COVID-19 more interpretable. Furthermore, we used a moving seven-day average to smoothen out short-term fluctuations and weekend effects for both, weather variables, cases lagged, and mobility variables (e.g. lower number of positive cases detected on weekends).

In summary, our mixed-effects model of the spread of the virus consists of the lagged cases per 100,000 capita for the $i$ municipalities, the government stringency, or the number of restrictions imposed on the Danish population. The model includes random intercepts for days in the time series and for the individual municipalities. This helps to account for time trends waves throughout the time series and differing spikes in incidence for individual municipalities. Furthermore, the $\mathrm{K}=2$ weather variables for the $i$ municipalities are included as well as the $L=2$ mobility changes, and the $M=2$ time-invariant demographics for the respective municipalities. Lastly, the $\mathrm{N}=5$ interactions are added to the model:

Equation (6):

$$
\begin{gathered}
\text { incidence }_{i, t}=\beta_{0}+\beta_{1} \text { incidence }_{i, t} \\
+\beta_{2} \text { stringency }_{t-9}+\sum_{k=3}^{K+2} \beta_{k}\left(\text { weather }_{k-2}\right)_{i, t-9}+\sum_{l=5}^{L+4} \beta_{l}\left(\text { mobility }_{l-4}\right)_{i, t-9} \\
+\sum_{m=7}^{M+6} \beta_{m}\left(\text { demographics }_{m-6}\right)_{i}+\sum_{n=9}^{N+8} \beta_{n}\left(\text { interactions }_{n-8}\right)_{i, t-9}
\end{gathered}
$$

\section{Data analysis}

Data preprocessing and statistical analyses were run using $R$ version 3.6.3 (R Core Team, 2021) in RStudio version 1.2.5033. Linear mixed effects models were run using the package Ime4 version 1.1-26 (Bates, Machler, Bolker \& Walker) and ImerTest version 3.1-3

(Kuznetsova, Brockhoff \& Christensen). Measures of Marginal- and Conditional $R^{2}$ are reported and calculated in accordance with (Nakagawa \& Schielzeth, 2013). Model 
comparison with a likelihood ratio test, to determine model fit, were done with anova version 3.6.2 (Chambers \& Hastie, 1992).

\section{Data and code availability}

The datasets generated and analysed during the current study are available in the github repository, https://github.com/Lassehhansen/COVID-19-Transmission. In the repository, information can be found on how to replicate analysis, preprocessing and plots. If issues occur during this process, an email for support is given within the repository.

\section{References}

Backer, J. A., Klinkenberg, D., \& Wallinga, J. (2020). Incubation period of 2019 novel coronavirus (2019-nCoV) infections among travellers from Wuhan, China, 20-28 January 2020. Euro Surveillance: Bulletin Europeen Sur Les Maladies Transmissibles = European Communicable Disease Bulletin, 25(5).

https://doi.org/10.2807/1560-7917.ES.2020.25.5.2000062

Badr, H. S., Du, H., Marshall, M., Dong, E., Squire, M. M., \& Gardner, L. M. (2020). Association between mobility patterns and COVID-19 transmission in the USA: a mathematical modelling study. The Lancet Infectious Diseases, 20(11), 1247-1254. https://doi.org/10.1016/S1473-3099(20)30553-3

Borkowski, P., Jażdżewska-Gutta, M., \& Szmelter-Jarosz, A. (2021). Lockdowned: Everyday mobility changes in response to COVID-19. Journal of Transport Geography, 90, 102906. https://doi.org/10.1016/j.jtrangeo.2020.102906

Chambers, J. M., \& Hastie, T. (1992). Statistical Models in S. Wadsworth \& Brooks/Cole Advanced Books \& Software. https://books.google.dk/books?id=uyfvAAAAMAAJ Chan, K. H., Peiris, J. S. M., Lam, S. Y., Poon, L. L. M., Yuen, K. Y., \& Seto, W. H. (2011a). The Effects of Temperature and Relative Humidity on the Viability of the SARS Coronavirus. Advances in Virology, 2011, 1-7. https://doi.org/10.1155/2011/734690

Chan, K. H., Peiris, J. S. M., Lam, S. Y., Poon, L. L. M., Yuen, K. Y., \& Seto, W. H. (2011b). 
The Effects of Temperature and Relative Humidity on the Viability of the SARS Coronavirus. Advances in Virology, 2011, 1-7. https://doi.org/10.1155/2011/734690

Chinazzi, M., Davis, J. T., Ajelli, M., Gioannini, C., Litvinova, M., Merler, S., Pastore y Piontti, A., Mu, K., Rossi, L., Sun, K., Viboud, C., Xiong, X., Yu, H., Halloran, M. E., Longini, I. M., \& Vespignani, A. (2020). The effect of travel restrictions on the spread of the 2019 novel coronavirus (COVID-19) outbreak. Science, 368(6489), 395-400. https://doi.org/10.1126/science.aba9757

Dergiades, T., Milas, C., \& Panagiotidis, T. (2020). Effectiveness of government policies in response to the COVID-19 outbreak. Available at SSRN 3602004.

DMI. (n.d.). Danish Meteorological Institute-Open Data. Retrieved 18 April 2021, from https://confluence.govcloud.dk/display/FDAPI/Danish+Meteorological+Institute+-+Op en+Data

Godzinski, A., \& Suarez Castillo, M. (2019). Short-term health effects of public transport disruptions: Air pollution and viral spread channels.

Google, L. (n.d.). Google COVID-19 Community Mobility Reports. Retrieved 18 April 2021, from https://www.google.com/covid19/mobility/

Hale, T., Angrist, N., Goldszmidt, R., Kira, B., Petherick, A., Phillips, T., Webster, S., Cameron-Blake, E., Hallas, L., Majumdar, S., \& Tatlow, H. (2021). A global panel database of pandemic policies (Oxford COVID-19 Government Response Tracker). Nature Human Behaviour, 5(4), 529-538. https://doi.org/10.1038/s41562-021-01079-8

Lowen, A. C., Mubareka, S., Steel, J., \& Palese, P. (2007). Influenza Virus Transmission Is Dependent on Relative Humidity and Temperature. PLoS Pathogens, 3(10), e151. https://doi.org/10.1371/journal.ppat.0030151

Nakagawa, S., \& Schielzeth, H. (2013). A general and simple method for obtaining R2 from generalized linear mixed-effects models. Methods in Ecology and Evolution, 4(2), $133-142$.

Nouvellet, P., Bhatia, S., Cori, A., Ainslie, K. E. C., Baguelin, M., Bhatt, S., Boonyasiri, A., 
Brazeau, N. F., Cattarino, L., Cooper, L. V., Coupland, H., Cucunuba, Z. M., Cuomo-Dannenburg, G., Dighe, A., Djaafara, B. A., Dorigatti, I., Eales, O. D., van Elsland, S. L., Nascimento, F. F., ... Donnelly, C. A. (2021). Reduction in mobility and COVID-19 transmission. Nature Communications, 12(1), 1090. https://doi.org/10.1038/s41467-021-21358-2

Pak, A., Adegboye, O. A., Adekunle, A. I., Rahman, K. M., McBryde, E. S., \& Eisen, D. P. (2020). Economic consequences of the COVID-19 outbreak: The need for epidemic preparedness. Frontiers in Public Health, 8.

Petersen, M. B., \& Roepstorff, A. (2021). Danskernes adfærd og holdninger til corona-epidemien. https://hope-project.dk/\#/reports/danskernes_adfaerd_og_holdninger

Qiu, Y., Chen, X., \& Shi, W. (2020). Impacts of Social and Economic Factors on the Transmission of Coronavirus Disease 2019 (COVID-19) in China [Preprint]. Epidemiology. https://doi.org/10.1101/2020.03.13.20035238

Rose, K. (n.d.). dkstat: API connection to the StatBank from Statistics Denmark [Github Page]. https://github.com/rOpenGov/dkstat

SSI, S. S. I. (n.d.). COVID-19 Dashboard. Retrieved 30 November 2021, from https://experience.arcgis.com/experience/aa41b29149f24e20a4007a0c4e13db1d?fb clid=IwAR3ZQCvyaY_YJmJ8Vt8xh5dsjluwJHNUKuABAyL5_dX7_x0xd9pNnFYvcal

Statistics Denmark. (2018). AREALDK: Land by land cover, region and unit. https://www.statistikbanken.dk/AREALDK

Statistics Denmark. (2020). BY2: Population 1. January by municipality, size of the city, age and sex. https://www.statistikbanken.dk/BY2

Varga, T. V., Bu, F., Dissing, A. S., Elsenburg, L. K., Bustamante, J. J. H., Matta, J., Zon, S. K. R. van, Brouwer, S., Bültmann, U., Fancourt, D., Hoeyer, K., Goldberg, M., Melchior, M., Strandberg-Larsen, K., Zins, M., Clotworthy, A., \& Rod, N. H. (2021). Loneliness, worries, anxiety, and precautionary behaviours in response to the COVID-19 pandemic: A longitudinal analysis of 200,000 Western and Northern 
Europeans. The Lancet Regional Health - Europe, 2, 100020.

https://doi.org/10.1016/j.lanepe.2020.100020

WHO. (2020). Coronavirus. https:// www.who.int/health-topics/coronavirus\#tab=tab_1

Yilmazkuday, H. (2020). Stay-at-home works to fight against COVID-19: International evidence from Google mobility data. Journal of Human Behavior in the Social Environment, 1-11.

\section{Author Contribution statement}

Lasse Hyldig Hansen and Thomas Lykke Rasmussen came up with the idea and conducted the analysis. Furthermore, Lasse Hyldig Hansen, Thomas Lykke Rasmussen, and Palle Villesen have all written the paper and the discussion of the results.

\section{Supplementary Figures}

Figure S1; Observed and predicted COVID-19 incidence (smoothed cases per 100,000 people) for all Danish municipalities in the period of analysis. Observed incidence (red line), Null model predictions (blue line). The grey shading around the lines is the 95\% confidence interval.

Figure S2. Conditional interaction plots of three interaction effects from the model. Figure a: the interaction effect of incidence lagged on incidence that depends on the stringency index. Figure b: the interaction effect of precipitation on incidence that depends on the stringency index. Figure c: the interaction effect of transit mobility change on incidence that depends on the stringency index. Incidence lagged, Precipitation and Transit Mobility Change are on a z-scale, and varies between -1 (1 SD below average) and 1 (1 SD above average). The same is the case for stringency, where 3 conditions of stringency are visualized (1 SD below average, average, and 1 SD above average). The $y$-variable is the conditional average COVID-19 incidence given the parameters of the model (predicted values given our model). 
Figures

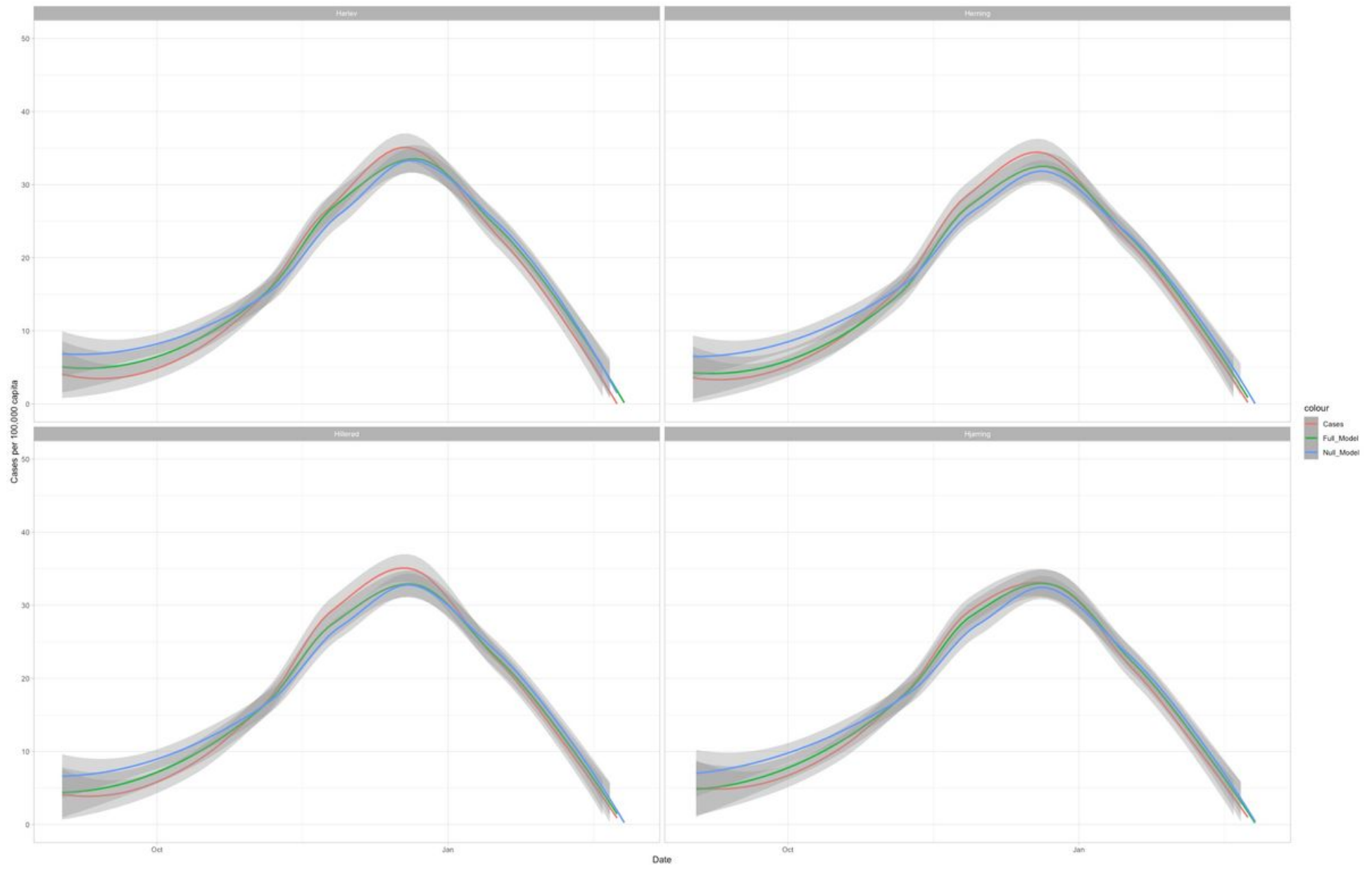

Figure 1

Observed and predicted COVID-19 incidence (smoothed cases per 100,000 people) for four Danish municipalities in the period of analysis. Observed incidence (red line), Null model predictions (blue line) and mixed effects model predictions (green line). The grey shading around the lines is the $95 \%$ confidence interval. 


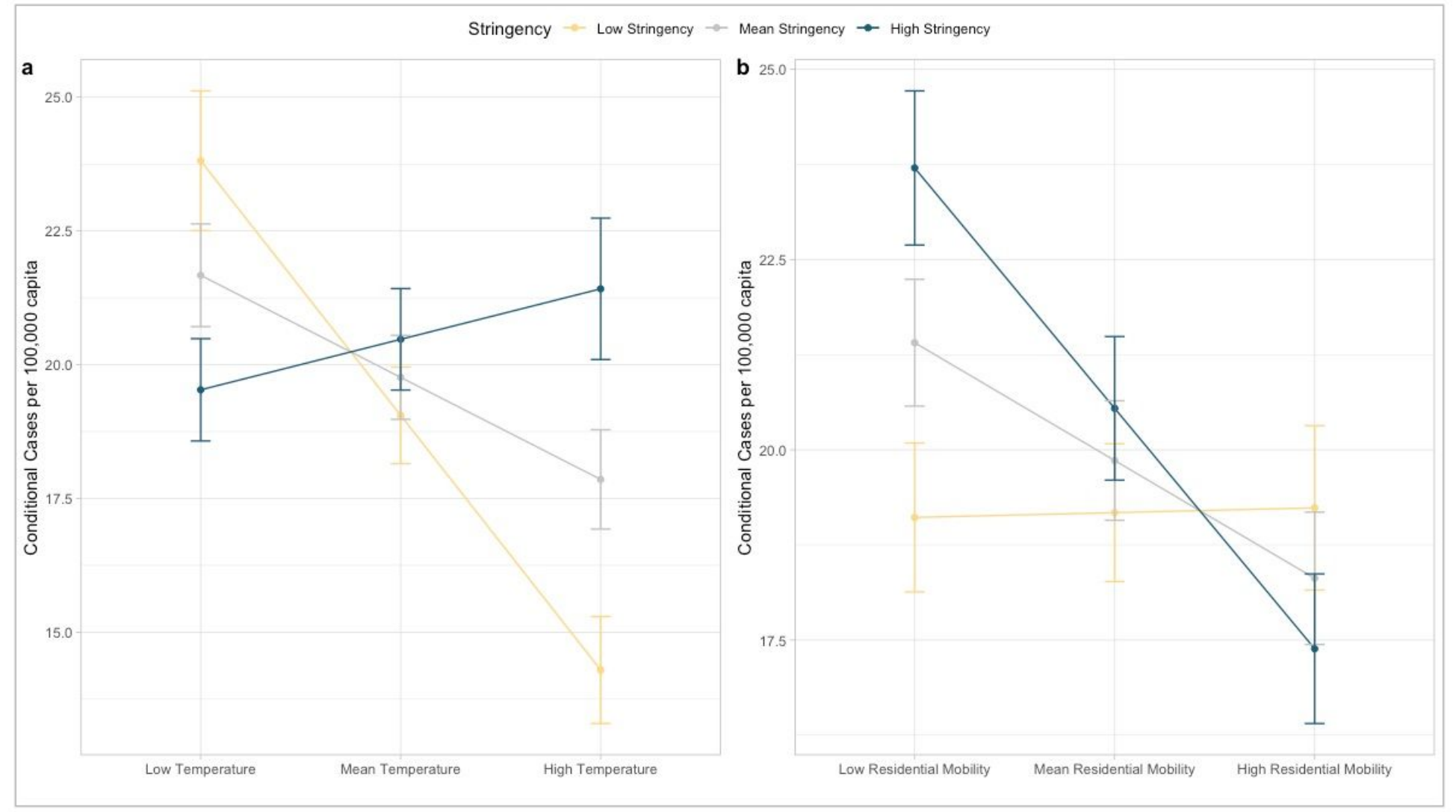

Figure 2

Conditional interaction plots of two of the interaction effects from the model. Figure a: the interaction effect of temperature on incidence that depends on the stringency index. Figure $b$ : the interaction effect of residential mobility change on incidence that depends on the stringency index. Temperature and residential mobility change are on a z-scale, and varies between -1 (1 SD below average) and 1 (1 SD above average). The same is the case for stringency, where 3 conditions of stringency are visualized ( 1 SD below average, average, and 1 SD above average). The y-variable is the conditional average COVID-19 incidence given the parameters of the model (predicted values given our model).

\section{Supplementary Files}

This is a list of supplementary files associated with this preprint. Click to download.

- FigureS1.jpeg

- FigureS2.jpeg 